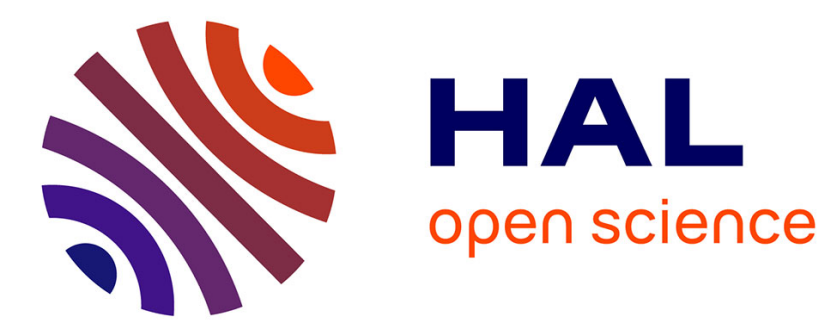

\title{
Cyclotron resonance in plasma flow
}

\author{
A. V. Artemyev, O. V. Agapitov, V. V. Krasnoselskikh
}

\section{To cite this version:}

A. V. Artemyev, O. V. Agapitov, V. V. Krasnoselskikh. Cyclotron resonance in plasma flow. Physics of Plasmas, 2013, 20 (12), pp.124502. 10.1063/1.4853615 . insu-01258001

\section{HAL Id: insu-01258001 https://hal-insu.archives-ouvertes.fr/insu-01258001}

Submitted on 18 Jan 2016

HAL is a multi-disciplinary open access archive for the deposit and dissemination of scientific research documents, whether they are published or not. The documents may come from teaching and research institutions in France or abroad, or from public or private research centers.
L'archive ouverte pluridisciplinaire HAL, est destinée au dépôt et à la diffusion de documents scientifiques de niveau recherche, publiés ou non, émanant des établissements d'enseignement et de recherche français ou étrangers, des laboratoires publics ou privés.

\section{(이) $\$$}

Distributed under a Creative Commons Attribution - NonCommercial - NoDerivatives| 4.0 


\section{AIP $\left.\right|_{\text {Physics of }}$

\section{Cyclotron resonance in plasma flow}

A. V. Artemyev, O. V. Agapitov, and V. V. Krasnoselskikh

Citation: Physics of Plasmas 20, 124502 (2013); doi: 10.1063/1.4853615

View online: http://dx.doi.org/10.1063/1.4853615

View Table of Contents: http://scitation.aip.org/content/aip/journal/pop/20/12?ver=pdfcov

Published by the AIP Publishing

\section{Articles you may be interested in}

Effects of non-Maxwellian species on ion cyclotron waves propagation and absorption in magnetically confined plasmas

Phys. Plasmas 12, 042508 (2005); 10.1063/1.1881472

Full-wave simulations using TORIC coupled to numeric MHD equilibrium solutions

AIP Conf. Proc. 595, 422 (2001); 10.1063/1.1424223

Ion cyclotron heating induced fast ion transport and plasma rotation in tokamaks

AIP Conf. Proc. 595, 385 (2001); 10.1063/1.1424214

Experimental evidence for spatial damping of left-hand circularly polarized waves in an electron cyclotron resonance region

Phys. Plasmas 8, 1455 (2001); 10.1063/1.1361256

Beam-plasma instability in inhomogeneous magnetic field and second order cyclotron resonance effects Phys. Plasmas 6, 692 (1999); 10.1063/1.873305

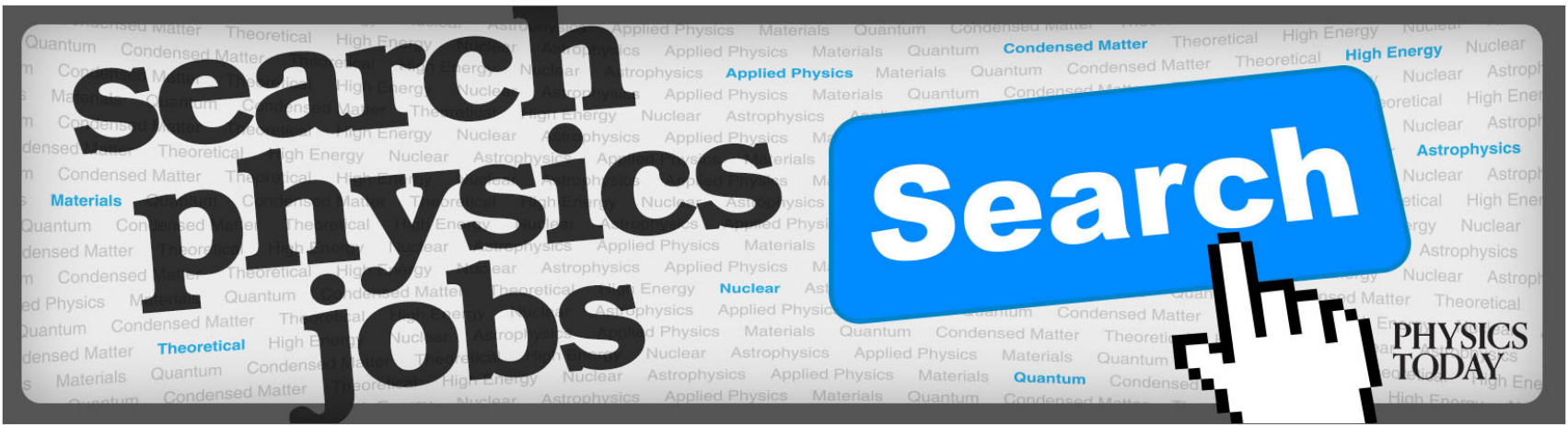




\title{
Cyclotron resonance in plasma flow
}

\author{
A. V. Artemyev, ${ }^{\text {a) }}$ O. V. Agapitov, ${ }^{\text {b) }}$ and V. V. Krasnoselskikh \\ LPC2E/CNRS-University of Orleans, Orleans, France
}

(Received 18 August 2013; accepted 9 December 2013; published online 23 December 2013)

This paper is devoted to the mechanism of particle acceleration via resonant interaction with the electromagnetic circular wave propagating along the inhomogeneous background magnetic field in the presence of a plasma flow. We consider the system where the plasma flow velocity is large enough to change the direction of wave propagation in the rest frame. This system mimics a magnetic field configuration typical for inner structure of a quasi-parallel shock wave. We consider conditions of gyroresonant interaction when the force corresponding to an inhomogeneity of the background magnetic field is compensated by the Lorentz force of the wave-magnetic field. The wave-amplitude is assumed to be about $10 \%$ of the background magnetic field. We show that particles can gain energy if $\mathbf{k v}_{s w}>\omega>\mathbf{k} \mathbf{v}_{s w}-\Omega_{c}$ where $\mathbf{k}$ is the wave number, $\mathbf{v}_{s w}$ is a plasma flow velocity, and $\omega$ and $\Omega_{c}$ are the wave frequency and the particle gyrofrequency, respectively. This mechanism of acceleration resembles the gyrosurfing mechanism, but the effect of the electrostatic field is replaced by the effect of the magnetic field inhomogeneity. (C) 2013 AIP Publishing LLC. [http://dx.doi.org/10.1063/1.4853615]

Wave-particle resonant interactions play an important role in magnetic energy dissipation and charged particle acceleration in space and astrophysical plasmas. There are two typical plasma configurations where efficient particle acceleration occurs. When a Lorentz force of a background magnetic field can be compensated for by forces due to action of a wave, a particle can be trapped in the wave effective potential. Two classical examples of such a system are the Landau damping ${ }^{1}$ and surfatron acceleration. ${ }^{2-4}$ When the background magnetic field is strong enough, particle motion is determined by Larmor rotation. For such a case, particle resonant acceleration is determined by cyclotron resonances ${ }^{5}$ and the magnetic field inhomogeneity plays a significant role. ${ }^{6}$

In a plasma without a magnetic field, the Cherenkov resonant condition $\mathbf{k v}=\omega$ plays a critical role in wave-particle interactions. For a strong magnetic field, this condition is replaced by the sum of the cyclotron resonances $k_{\|} v_{\|}$ $=\omega-n \Omega_{c}$ where $\mathbf{v}$ and $\mathbf{k}$ are the particle velocity and wavenumber $\left(v_{\|}, k_{\|}\right.$are components along the background magnetic field), $\omega$ is a wave frequency, and $\Omega_{c}$ is a cyclotron frequency in the background magnetic field $(n=0, \pm 1, \pm 2$ is the resonance number). Classical Landau resonance $n=0$ corresponds to a particle motion with the same direction as $\mathbf{k}$ $\left(k_{\|} v_{\|}=\omega>0\right)$ and can be realized for waves with a strongly oblique propagation relative to the background magnetic field. ${ }^{6,7}$ For almost parallel waves, the first (or fundamental) cyclotron resonance $n=1$ is the most important. ${ }^{6,8}$ For this case, particles should move in opposite directions with $\mathbf{k}$ to be in the resonance $k_{\|} v_{\|}=\omega-\Omega_{c}<0$ for $\omega<\Omega_{c}$. Thus, particle resonant interactions with a finite size wave-packet are bounded in time, and, as a result, the efficiency of such an acceleration is limited. ${ }^{9}$

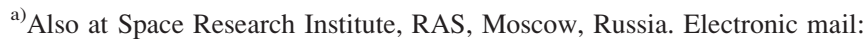
ante0226@gmail.com.

b) Also at Space Sciences Laboratory, University of California, Berkeley, California, USA and at National Taras Shevchenko University of Kiev, Kiev, Ukraine.
}

Here, we consider a system where the first cyclotron resonance can be realized for particles moving with the same direction as $\mathbf{k}$. Such a condition is possible if the system contains rapid plasma flow in a direction opposite to $\mathbf{k}$. Corresponding conditions can be found for regions of solar wind interactions with quasi-parallel shock waves. Such shock waves contain numerous small-scale structures characterized by the increased strength of the magnetic field, with strong wave activity within the vicinity of them. ${ }^{10-12}$ Waves propagating away from these structures often have a phase velocity smaller than the solar wind velocity and, as a result, these waves move in an opposite direction than that of their wave-vectors (see scheme in Fig. 1). In such a case, specific conditions for particle acceleration can be realized.

To describe wave-particle resonant interactions, we introduce a simplified model of the external magnetic field $\mathbf{B}_{0}=B_{z}(x) \mathbf{e}_{z}+B_{x} \mathbf{e}_{x}$ (see scheme in Fig. 1) with $B_{z}(x)$ $=B_{z 0}=$ const for $x<0$. We also introduce the constant parameter $b_{z}=B_{z 0} / B_{x}$ and the function $\nu(x)=B_{z}(x) / B_{z 0}$. In the lab frame, supposed to be associated with the background inhomogeneous magnetic field, the plasma (solar wind) flows with a velocity $\mathbf{v}_{s w}=v_{\text {flow }} / \nu(x) \mathbf{e}_{x}$ (i.e., $\mathbf{v}_{s w} \times \mathbf{B}_{0}$ $=$ const ). We consider a circular wave propagating along the magnetic field, but in the direction opposite to the magnetic field gradient (i.e., in the lab frame the wave phase is $\phi=-\int k_{\|}(s) d s-\omega t$, where $s$ is a coordinate along the magnetic field). The solar wind velocity, $\mathbf{v}_{s w}$ can be taken into account using transformation $\omega \rightarrow \omega-\mathbf{k v}_{s w}<0$. Through the paper we consider particle motion in the lab frame with the shifted wave frequency. Furthermore, we use $\Omega_{w}(t)$ $=\mathbf{k v}_{s w}-\omega>0$ and $\phi=-\left(\int k_{\|}(s) d s-\int \Omega_{w}(t) d t\right) \quad\left(\Omega_{w}(t)\right.$ depends on $t$ due to a spatial inhomogeneity of the solar-wind velocity and wave number). To simplify all estimates and calculations, we assume the absence of the transverse component of the wave number $\left(\mathbf{k} \times \mathbf{B}_{0}=0\right)$. Thus, we have following relation between components of the wave number, 


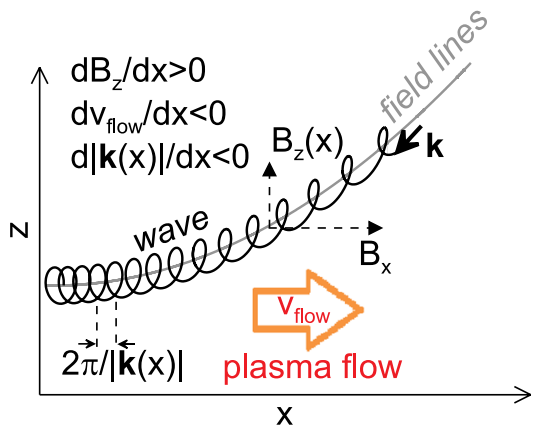

FIG. 1. Schematic view of the system.

$\mathbf{k}=k_{x}(x) \mathbf{e}_{x}+k_{z} \mathbf{e}_{z}: \quad k_{x}(x)=k_{z} B_{x} / B_{z}(x)=k_{z} / \nu(x) b_{z} . \quad \mathrm{We}$ introduce constant parameter $k=k_{z} / b_{z}$ and rewrite the following wave phase $\phi \rightarrow-\phi$ as $\phi=k b_{z} z+k \int d x / \nu(x)$ $-\int \Omega_{w}(t) d t$.

The magnetic field of the circular right-hand polarized wave (e.g., the magnetosonic wave often observed near a shock-wave, see Ref. 17) can be written, as follows:

$$
\mathbf{B}_{\delta}=B_{\delta} b_{z} \cos \phi \mathbf{e}_{x}+B_{\delta}(b / \nu) \sin \phi \mathbf{e}_{y}-B_{\delta} \nu^{-1} \cos \phi \mathbf{e}_{z},
$$

where $B_{\delta}$ is the wave amplitude and $b(x)=\sqrt{1+b_{z}^{2} \nu^{2}(x)}$. For $\mathbf{B}_{\delta}$, we have $\mathbf{B}_{\delta} \cdot \mathbf{B}_{0}=0$, and the magnitude $\left|\mathbf{B}_{\delta}\right|^{2}=B_{\delta}^{2}(b / \nu)^{2}$ does not depend on the wave phase (as it should be for circular waves), but depends on $x$. The electric field of the wave can be determined from the Maxwell equations, as follows:

$$
\mathbf{E} / B_{\delta}=\frac{v_{\phi}}{c}\left(b_{z} \sin \phi \mathbf{e}_{x}-\frac{b}{\nu} \cos \phi \mathbf{e}_{y}-\frac{1}{\nu} \sin \phi \mathbf{e}_{z}\right),
$$

where $v_{\phi}=\left(\Omega_{w}(t) / k\right) \nu / b$.

Equations of motion for a nonrelativistic ion with a charge $q$ and a mass $m$ in the system with a magnetic field $\mathbf{B}_{0}+\mathbf{B}_{\delta}$ and the electric field $\mathbf{E}_{\delta}$ can be written as

$$
\begin{aligned}
& \dot{v}_{x}=\Omega_{0} \nu b_{z} v_{y}-\Omega_{\delta} \frac{1}{\nu} v_{y} \cos \phi-\left(v_{z}-\frac{v_{\phi} b_{z} \nu}{b}\right) \Omega_{\delta} \frac{b}{\nu} \sin \phi \\
& \dot{v}_{z}=-\Omega_{0} v_{y}-\Omega_{\delta} b_{z} v_{y} \cos \phi+\left(v_{x}-\frac{v_{\phi}}{b}\right) \Omega_{\delta} \frac{b}{\nu} \sin \phi \\
& \dot{v}_{y}=-\Omega_{0}\left(v_{x} b_{z} \nu-v_{z}\right)+\left(v_{z} b_{z}+v_{x} \frac{1}{\nu}-v_{\phi} \frac{b}{\nu}\right) \Omega_{\delta} \cos \phi \\
& \dot{\phi}=k\left(b_{z} v_{z}+v_{x} / \nu\right)-\Omega_{w}(t),
\end{aligned}
$$

where $\Omega_{0}=q B_{x} / m c=$ const and $\Omega_{\delta}=q B_{\delta} / m c=$ const . We introduce the parallel velocity $v_{\|}=\left(v_{z} b_{z} \nu+v_{x}\right) / b$, the amplitude of the perpendicular velocity $v_{\perp}$ and the corresponding phase $\theta: v_{\perp} \cos \theta=\left(v_{z}-v_{x} b_{z} \nu\right) / b$ and $v_{\perp} \sin \theta=v_{y}$. With new variables, Eqs. (1) take the following form:

$$
\left\{\begin{array}{l}
\dot{v}_{\|}=-\Omega_{\delta} \frac{b}{\nu} v_{\perp} \sin \Delta \phi+\frac{b_{z} \dot{\nu}}{b^{2}} v_{\perp} \cos \theta \\
\dot{v}_{\perp}=-\Omega_{\delta} \frac{b}{\nu}\left(v_{\phi}-v_{\|}\right) \sin \Delta \phi-\frac{b_{z} \dot{\nu}}{b^{2}} v_{\|} \cos \theta \\
\dot{\theta}=-\Omega_{0} b-\Omega_{\delta} \frac{b v_{\|}-v_{\phi}}{\nu} \cos \Delta \phi+\frac{b_{z} \dot{\nu}}{b^{2}} \frac{v_{\|}}{v_{\perp}} \sin \theta \\
\dot{\phi}=(b k / \nu)\left(v_{\|}-v_{\phi}\right),
\end{array}\right.
$$

where $\Delta \phi=\phi-\theta$. We assume that the background magnetic field is strong enough $\left(\Omega_{0} b \gg \dot{\nu}, \Omega_{0} b \gg \Omega_{\delta}\right)$ to use the approximation $\dot{\theta} \approx-\Omega_{0} b$. This approximation is taken into account only for analytical estimates. The resonant condition corresponds to the existence of a stationary point of the phase difference $\Delta \phi$. The corresponding equation $\Delta \dot{\phi}=0$ can be written as follows: $v_{\|}-v_{\phi}+\left(\nu \Omega_{0} / k\right)=0$. Terms with a multiplier $\sim \cos \theta$ are neglected, because $\theta$ is a fast oscillating variable. Therefore, in the vicinity of the resonance $\Delta \dot{\phi} \approx 0$, Eqs. (2) can be rewritten as

$$
\begin{aligned}
& \frac{d v_{\|}^{2}}{d \nu}=-\frac{4 \Omega_{\delta} b^{2} v_{\perp}}{\left(d \nu^{2} / d x\right)} \sin \Delta \phi, \\
& \frac{d v^{2}}{d t}=-2 \Omega_{\delta} \frac{b}{\nu} v_{\phi} v_{\perp} \sin \Delta \phi,
\end{aligned}
$$

where $v^{2}=v_{\|}^{2}+v_{\perp}^{2}, v_{\|}=v_{\phi}-\nu \Omega_{0} / k$, and $v_{x}=\left(v_{\|}-v_{\perp} \cos \theta\right)$ $\approx v_{\|} / b$. The first equation provides the condition of resonance while the second equation provides the variation of the total energy, $v^{2}$. A combination of these two equations gives

$$
\frac{d v^{2}}{d t}=\frac{v_{\phi}}{2 b \nu} \frac{d \nu^{2}}{d x} \frac{d v_{\|}^{2}}{d \nu}
$$

The gradient $d \nu^{2} / d x$ is positive. Therefore, the growth of the total energy is given by the inequality $v_{\phi} v_{\|}\left(d v_{\|} / d \nu\right)>0$, which can be rewritten as follows:

$$
v_{\phi} v_{\|}\left(-\frac{v_{\text {flow }}\left(2 b^{2}-1\right)}{\nu^{2}}-\frac{\omega+\Omega_{0} b^{3}}{k}\right)>0 .
$$

Here we use the definition of $v_{\|}$

$$
v_{\|}=v_{\phi}-\frac{\nu \Omega_{0}}{k}=\frac{\nu}{b}\left(\frac{v_{\text {flow }}}{\nu^{2}}-\frac{\omega+\Omega_{0} b}{k}\right) .
$$

The last term of Eq. (5) $\sim d v_{\|} / d \nu$ is always negative because $b>1$. Therefore, we have the following condition for particle acceleration $v_{\phi} v_{\|}<0$, which can be rewritten as an inequality for $\nu$ :

$$
\frac{k v_{\text {flow }}}{\omega}>\nu^{2}>\frac{k v_{\text {flow }}}{\omega}\left(1+\frac{\Omega_{0} b}{\omega}\right)^{-1},
$$

where $b$ depends on $\nu$. For the initial variables the inequalities can be written as $\omega<\mathbf{k v}_{s w}$ and $\omega>\mathbf{k v}_{s w}-\Omega_{c}$, where $\Omega_{c}(x)=\Omega_{0} b(x)$. The Doppler shift should be larger than the wave-frequency, but smaller than the sum of the wave frequency and the gyrofrequency.

The complicate expressions for $\dot{v}_{\|}$and $\dot{v}_{\perp}$ cannot be used to derive a final analytical equation for the energy evolution in the resonance. Therefore, we numerically integrate system (1). For this reason, we introduce the simple function $\nu(x)=1+x / L_{x}$, where $L_{x}$ is the spatial scale of the magnetic field inhomogeneity. We assume that $\nu(x)=1$ for $x<0$. We use the dimensionless parameters $\ell=L_{x} q B_{0} /$ $m c v_{\text {flow }}, \kappa=k v_{\text {flow }} m c / q B_{0}$. For all of the calculations, we use $b_{z}=0.1$ and $B_{\delta} / B_{0}=0.1$. 
Numerical solutions for system (1) for various system parameters are shown in Fig. 2. The top panels demonstrate the growth of energy $v^{2}$ with time. The energy $v^{2}$ oscillates and increases by small jumps. Each jump directly relates to passage through the resonance $\Delta \dot{\phi}=0$ where we use $\dot{\theta} \approx-b \Omega_{0}$ to plot $\Delta \dot{\phi}$ in the figure (for numerical calculations this approximation is not used). Trajectories of particles in the velocity plane $\left(v_{\perp} \sin \theta, v_{\perp} \cos \theta\right)$ perpendicular to the magnetic field resemble a spiral (i.e., the increase in energy results in the unwinding of this spiral - the growth of corresponding radius of the spiral). Particles escape from the resonance as soon as the second inequality in Eq. (7) is violated. Therefore, our estimates for the parametric region of the resonance interaction are correct. Due to inequality (7), the resonant velocity $v_{\|}=v_{\phi}-\nu \Omega_{0} / k$ should be negative. From Eq. (6), the amplitude of an initial velocity in the lab frame can be estimated as follows:

$$
\frac{v_{0 x}}{v_{\text {flow }}} \approx \frac{v_{\|}}{b v_{\text {flow }}} \approx-\frac{1}{\nu}\left(\frac{\nu^{2}}{\kappa b}\left(1+\frac{\omega}{\Omega_{0} b}\right)-\frac{1}{b^{2}}\right) .
$$

Due to $b_{z} \ll 1$ one can use $b \approx 1$ and obtain $v_{x 0} / v_{\text {flow }}$ $\sim 0.1-0.3$ for $\kappa \sim 3-5$ and $\nu \sim 1.5-2$. Therefore, resonant
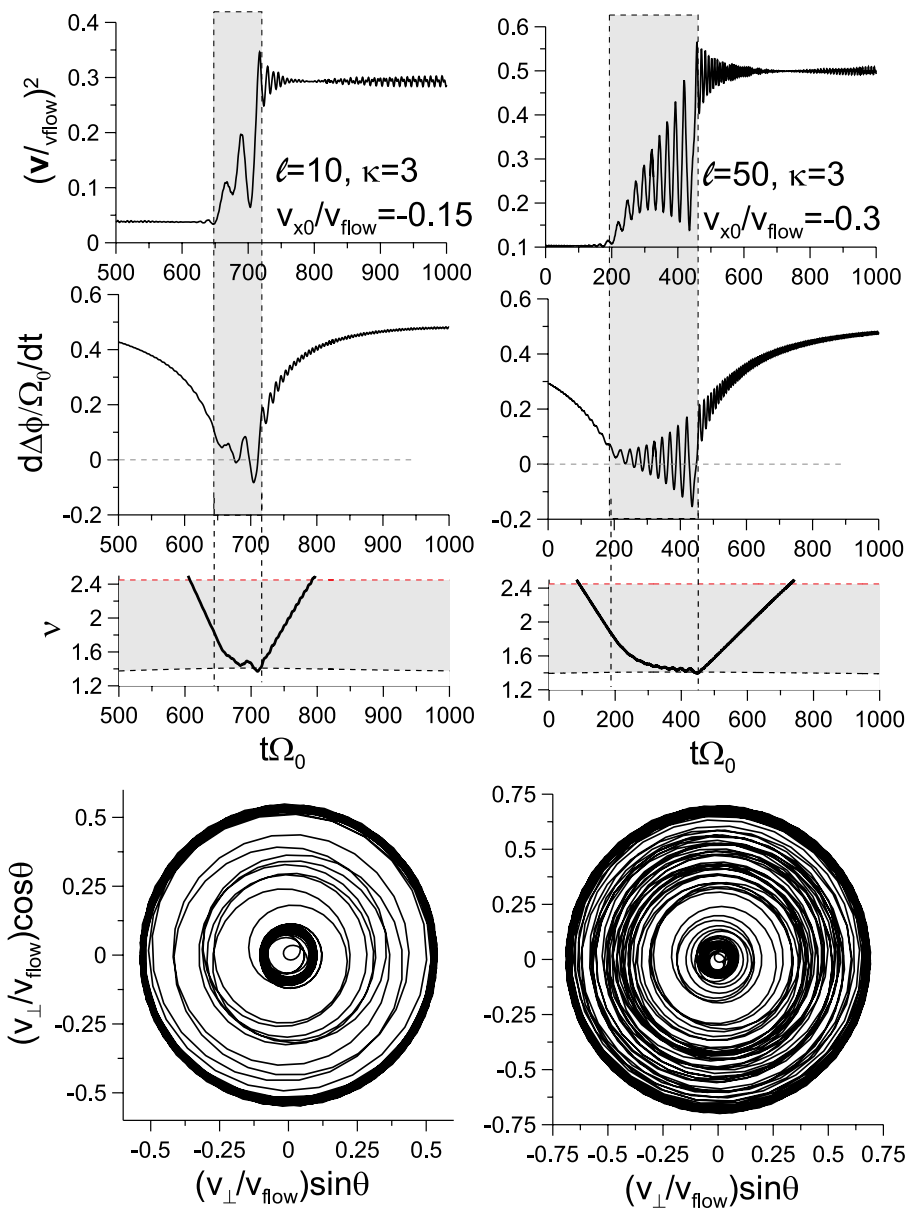

particles correspond to initial energy of $\sim 101 \%-110 \%$ of the energy of plasma flow.

We also find that particles can resonantly interact several times with the wave (see Fig. 3). A particle should have an initially negative $v_{\|}$and move toward the decrease of $\nu$. Following the first resonant interaction with the wave, the particle gains some energy. Resonant interaction with energy growth corresponds to the growth of $v_{\|}$(i.e., $\left|v_{\|}\right|$decreases). Therefore, resonance can result in a change of $v_{\|}$sign. The particle turns round and begin moving with $v_{\|}>0$ toward the increase of $\nu$ (an increase of $b$ ). Due to the mirror force, this particle after a certain time should turn round and begin moving with $v_{\|}<0$ again. For this case, the second resonant interaction is possible, etc. The example shown in Fig. 3 demonstrates that the particle gains a large energy $v^{2}>$ $4 v_{\text {flow }}^{2}$ due to several resonant interactions. Eventually, the particle leaves the region with the magnetic field gradient (in the Fig. 3 this moment is called reflection).

Now, we compare our results with previous estimates obtained for cyclotron resonant acceleration in the absence of plasma flow. ${ }^{14-16} \mathrm{We}$ consider the particle acceleration in the gyroresonance with an electromagnetic circular wave propagating parallel to the magnetic field. For this system, the equation for parallel velocity $v_{\|}$takes the form
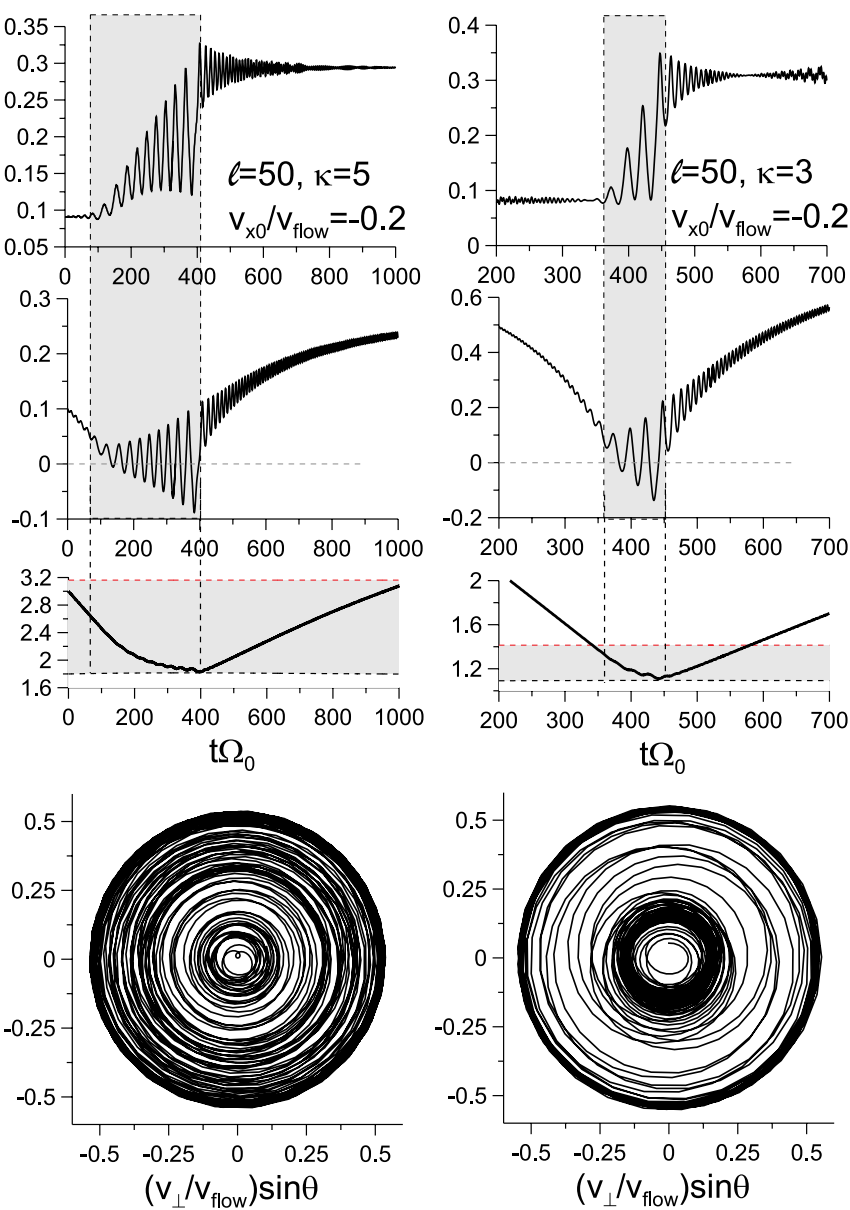

FIG. 2. Results of the numerical integration of system (1). The top panels show particle energies as functions of time. The middle panels show $\Delta \dot{\phi} / \Omega_{0}$ (with $\left.\theta=-\Omega_{0} b\right)$ and $\nu$ along the trace. The bottom panels show particle trajectories in the velocity plane $\left(v_{\perp} \cos \theta, v_{\perp} \sin \theta\right)$. In panels with $\nu$ the horizontal curves show $\sqrt{k v_{\text {flow }} / \omega}$ (top) and $\sqrt{k v_{\text {flow }} / \omega}\left(1+\Omega_{0} b / \omega\right)^{-1 / 2}$ (bottom) where $b$ depends on $\nu$. For three first cases, the wave frequency is $\omega / \Omega_{0}=0.5$, while for the last (the right most) case $\omega / \Omega_{0}=1.5$. The grey color indicates the time interval of the particle resonant acceleration. The initial velocity of particles in the $x$-direction is indicated inside top panels. 

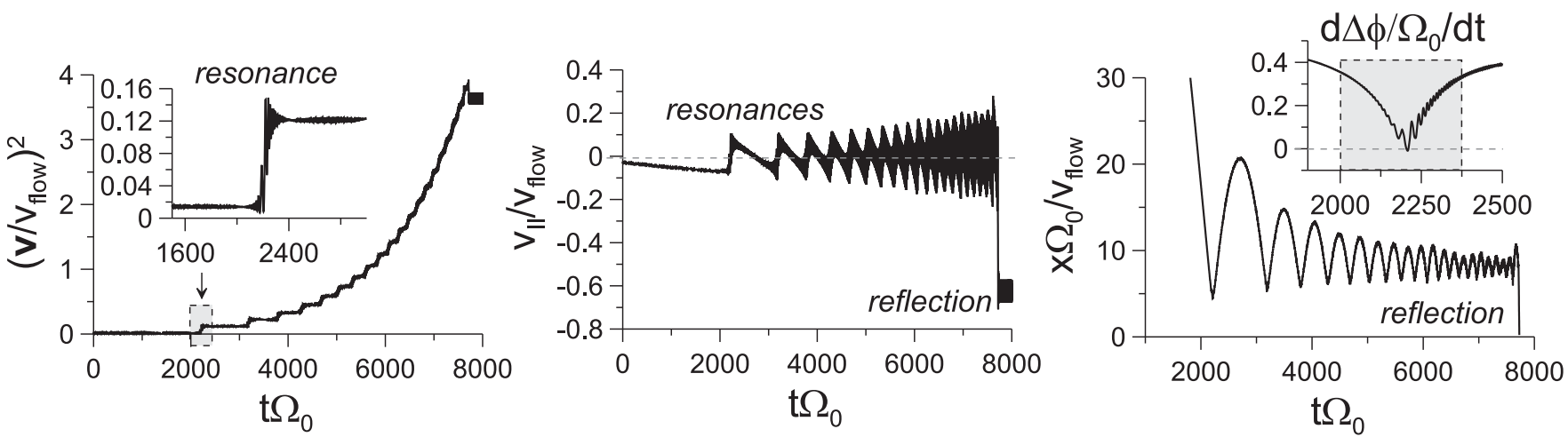

FIG. 3. The trajectory of a particle with several resonant interactions. The first interaction is shown in the grey box.

$$
\dot{v}_{\|} \approx-\Omega_{\delta} v_{\perp}(b / \nu) \sin \Delta \phi
$$

The resonant condition $\Delta \dot{\phi}=0$ requires that the Lorentz force of the wave $\Omega_{\delta} v_{\perp}(b / \nu) \sin \phi_{0}$ should be balanced by the inhomogeneity of the system $\sim \dot{v}_{\|} \approx(1 / 2 b) d v_{\|}^{2} / d x$. In this case, the equation for the total energy (4) can be written, as follows:

$$
d v^{2} / d t=d v_{\phi}^{2} / d t-\left(2 \Omega_{w} / k_{z}^{2}\right) \dot{\Omega}_{c}
$$

This is the simplified form of the well-known equation for the first order cyclotron resonance of particles with a wave in the inhomogeneous magnetic field (e.g., Ref. 6). For a case of an absence of plasma flow $\Omega_{w}=-\omega$, one can easily obtain $d v^{2} / d t \sim \dot{\Omega}_{c}$ (i.e., the gain of energy is due to particle motion in the direction of the magnetic field increase-the so-called betatron mechanism of acceleration). The corresponding parallel velocity is $v_{\|} \sim-\left(\omega+\Omega_{c}\right)<0$ and the energy growth $d v^{2} / d t \sim\left(d \Omega_{c} / d s\right) v_{\|}$is possible only in a system with $d \Omega_{c} / d s<0$ (for right-handed polarized waves). In this paper, we consider the case of a sufficiently strong plasma flow when particles can be accelerated due to $d v^{2} / d t \sim-\dot{\Omega}_{c}>0$ with $v_{\|}<0$ and $d \Omega_{c} / d s>0$.

An alternative possibility of compensation of the wave Lorentz force was proposed in Ref. 13 where the background electric field $E_{\|}$was introduced. For this case, one can use the homogeneous magnetic field $b=1$ and write the equation for $v_{\|}$as

$$
\dot{v}_{\|} \approx \Omega_{\delta} w_{\|}-\Omega_{\delta} v_{\perp} \sin \Delta \phi
$$

where $w_{\|}=c E_{\|} / B_{\delta}$. The condition of the gyroresonance $v_{\|}=\dot{\phi} / k+v_{\phi}\left(\right.$ and $\left.\dot{v}_{\|}=0\right)$ can be satisfied if $w_{\|} \approx v_{\perp}$ $\sin \Delta \phi$. The corresponding equation for $v_{\perp}$ has the form $k \dot{v}_{\perp} \approx-w_{\|} / v_{\perp}$. Therefore, for $w_{\|}<0$ the energy of a particle increases $d v_{\perp}^{2} / d t>0$ (see Ref. 13).

In conclusion, in this work, we consider the particle resonant interaction with circular electromagnetic waves when the background magnetic field is non-homogeneous.
The presence of a rapid plasma flow allows one to shift the wave-frequency, resulting in the acceleration of particles moving with the same direction as $\mathbf{k}$. The effect is similar to that described in Ref. 13 , but does not require the presence of the additional electrostatic field that was not observed around small-scale magnetic field structures in quasi-parallel shocks (see Ref. 17). For our mechanism, an impact similar to this action is provided by the natural magnetic field inhomogeneity.

The study was supported by the Grant of CNES "Cluster Co-I of DWP'. Work of A.V.A. was also partially supported by Russian Academy of Science (OFN-15).

${ }^{1}$ L. D. Landau, Sov. JETP 16, 574 (1946).

${ }^{2}$ R. Z. Sagdeev and V. D. Shapiro, Sov. JETP Lett. 17, 279-282 (1973).

${ }^{3}$ T. Katsouleas and J. M. Dawson, Phys. Rev. Lett. 51, 392-395 (1983).

${ }^{4}$ A. I. Neishtadt, A. V. Artemyev, L. M. Zelenyi, and D. L. Vainshtein, JETP Lett. 89, 441-447 (2009).

${ }^{5}$ A. Timofeev, Resonance Phenomena in Plasma Oscillation (Taylor \& Francis Group, 2010).

${ }^{6}$ D. Shklyar and H. Matsumoto, Surv. Geophys. 30, 55-104 (2009).

${ }^{7}$ A. Artemyev, V. Krasnoselskikh, O. Agapitov, D. Mourenas, and G. Rolland, Phys. Plasmas 19, 122901 (2012).

${ }^{8}$ V. Y. Trakhtengerts and M. J. Rycroft, Whistler and Alfvén Mode Cyclotron Masers in Space (Cambridge University Press, 2008).

${ }^{9}$ X. Tao, J. Bortnik, R. M. Thorne, J. M. Albert, and W. Li, Geophys. Res. Lett. 39, L06102, doi:10.1029/2012GL051202 (2012).

${ }^{10}$ S. J. Schwartz and D. Burgess, Geophys. Res. Lett. 18, 373-376, doi:10.1029/91GL00138 (1991).

${ }^{11}$ L. B. Wilson, A. Koval, D. G. Sibeck, A. Szabo, C. A. Cattell, J. C. Kasper, B. A. Maruca, M. Pulupa, C. S. Salem, and M. Wilber, J. Geophys. Res. 118, 957-966, doi:10.1029/2012JA018186 (2013).

${ }^{12}$ D. Burgess and M. Scholer, Space Sci. Rev. 178, 513-533 (2013).

${ }^{13}$ Y. Kuramitsu and V. Krasnoselskikh, Phys. Rev. Lett. 94, 031102 (2005).

${ }^{14}$ D. R. Shklyar, Sov. JETP 53, 1197-1192 (1981).

${ }^{15}$ V. Y. Trakhtengerts, M. J. Rycroft, D. Nunn, and A. G. Demekhov, J. Geophys. Res. 108, 1138, doi:10.1029/2002JA009559 (2003).

${ }^{16}$ J. Bortnik, R. M. Thorne, and U. S. Inan, Geophys. Res. Lett. 35, L21102, doi:10.1029/2008GL035500 (2008).

${ }^{17}$ A. Kis, O. Agapitov, V. Krasnoselskikh, Y. V. Khotyaintsev, I. Dandouras, I. Lemperger, and V. Wesztergom, Astrophys. J. 771, 4 (2013). 